\title{
Quadrivalent Human Papillomavirus Vaccine and the Young Ovary: Review of Safety Research Following Two Case Series of Premature Ovarian Insufficiency
}

\section{Little D*}

Department of General Practice, Bellingen District Hospital, Bellingen, Australia

*Corresponding author: Little D, MBBS DRANZCOG FACRRM, Bellingen District Hospital, Church Street, Bellingen, New South Wales 2454, Australia, E-mail: dlittle@skymesh.com.au

Citation: Little D (2017) Quadrivalent Human Papillomavirus Vaccine and the Young Ovary: Review of Safety Research Following Two Case Series of Premature Ovarian Insufficiency. J Immunol Infect Dis 4(1): 101

\section{Received Date: January 26, 2017 Accepted Date: June 13, 2017 Published Date: June 15, 2017}

\begin{abstract}
The quadrivalent virus-like particle vaccine against human papillomavirus (QHPV) is a population vaccine targeting peri-pubertal girls and boys. Focus on evaluation of adolescent ovarian safety arises from cases in primary care and published case series describing the development of menstrual dysfunction and idiopathic premature ovarian insufficiency (POI) following QHPV vaccination. A structured review of QHPV vaccine safety studies was performed to answer the question 'has the ongoing ovarian safety of this vaccine in adolescents been assessed?' The design, size and process of pre-licensing safety trials in relation to their capacity to assess ovarian function were reviewed. A structured literature search and review of post-licensing QHPV vaccine safety studies was conducted to September 2015. Post-licensing safety studies were hand searched for adolescent enrolment. These studies were further searched for documentation of menstrual function and ability to detect abnormal menstrual function. Vaccine adverse event notifications and regulatory safety statements were sought. Pre-licensing controlled safety trials did not investigate ovarian function. Capacity to observe ovarian function was impeded by safety trials' design, size and reporting process. Both placebo controls were comprised of vaccine constituents. Both were incorrectly identified. No controlled safety studies post-marketing reported on ovarian function or had the capacity to do so due to their design and pre-specified objectives. One small observational research finding from a syncope unit suggests new menstrual dysfunction in $48 \%$ of vaccinated young women. The ongoing ovarian safety of this vaccine in adolescents has not been assessed.
\end{abstract}

Keywords: Premature ovarian failure; Premature menopause; Gardasil ${ }^{\oplus}$; Premature ovarian insufficiency

\section{Introduction}

QHPV vaccine is a new, recombinant protein particulate vaccine, containing 20, 40, 40, $20 \mu \mathrm{g}$ of the major capsid (L1) protein of HPV types 6, 11, 16, and 18 respectively, $225 \mu \mathrm{g}$ aluminium hydroxyphosphate sulphate, $9.65 \mathrm{mg}$ sodium chloride, $780 \mu \mathrm{g}$; L-histidine, $50 \mu \mathrm{g}$ polysorbate 80 , and $35 \mu \mathrm{g}$ sodium borate ('GARDASIL ${ }^{\circledast}$ ' $\mathrm{HPV} 4$ ', '4vHPV') in each of three doses. Its protective role against the most common human papillomavirus (HPV) oncogenic types, HPV 16 and 18, can prevent 70\% of cervical cancer [1]. QHPV vaccination commenced in Australia in 2007 and the National Immunization Programme targets 12-13 year-olds [2] to receive three dosages of this vaccine. In the USA QHPV vaccination at age 11-12 years has been recommended by the Advisory Committee on Immunization Practices (ACIP) since 2006 for females as a three dose regime, with extension of immunization offered to age 26 years.

Recent published case series of young adolescents with established diagnoses of idiopathic premature menopause [3-5] (also known as premature ovarian failure (POF) and premature ovarian insufficiency (POI) more appropriately) arising in QHPV-vaccinated girls have been described in several medical journals [3-5] and presented at three international congresses [6,7]. It cannot be known whether there may be a causative link between these case series and QHPV vaccine. Aberrant ovarian function cannot be ascribed to this vaccine without appropriate research. This article presents the latest paper delivered to the 10th International Congress on Autoimmunity, Leipzig April 2016. Close attention to this vaccine's safety research is warranted by such series and their potentially devastating impact. POI is defined as hypergonadotropic hypogonadism arising before age 40 due to follicle depletion or dysfunction [8]. In two case series of six established idiopathic POI diagnoses, one series from an Australian medical practice, four girls (aged 14, 14, 15 and 21) developed POI symptoms (irregular periods, oligomenorrhoea and amenorrhoea) within 12 months of third vaccination. A fifth girl was using the oral contraceptive pill (OCP) when fully vaccinated at age 13 , and was amenorrhoeic at OCP cessation age 14. The OCP was recommenced for 4 years. After OCP cessation amenorrhoea resumed at 
age 18. The sixth girl was vaccinated pre-menarche. Menarche at age 15 was followed by one menstruation before amenorrhoea. All girls were subsequently diagnosed with idiopathic premature ovarian insufficiency [3-5]. This review investigates whether existing research can attest to ovarian safety in adolescents post-vaccine.

It is known that $92 \%$ of women with POF describe an altered menstrual cycle as the initial symptom [9]. Presenting symptoms include oligomenorrhoea, polymenorrhoea, metrorrhagia and vasomotor symptoms. 58\% describe amenorrhoea lasting 3 months, but only $39 \%$ of women developing amenorrhoea consult a doctor [10]. 25\% of POF takes more than 5 years to diagnose from onset of cycle disturbance [9] and diagnosis mostly requires 3 clinician visits before laboratory testing. Girls presenting with these symptoms in the Australian series [4,5] had all been prescribed oral contraception for 'cycle regulation', delaying diagnosis.

Due to the rarity of idiopathic premature menopause in young adolescence, there are no figures for background age-specific incidence for comparison. The annual incidence of 'premature menopause' under 30 years of age is reported as one in 10/100,000 person years [11]. Absent age-specific comparators increase the need to evaluate the safety research capacity to identify POI. Rare, new-onset medical conditions are less detectable in baseline safety studies. Additional design limitations and weaknesses could further reduce their capacity to attest to ovarian safety [12].

\section{Method}

A structured safety review evaluated evidenced ovarian integrity and function post QHPV vaccine to answer the question: 'Has the ongoing ovarian safety of this vaccine in young adolescents been assessed?'.

Investigation reviewed pre-licensing and post-licensing studies. Pre-licensing studies included toxicology studies and clinical safety trials identified at licensing by the Vaccine and Related Biological Products Advisory Committee (VRBPAC) to the Food and Drug Administration (FDA) USA Licensing Application. Post-licensing safety studies included research meeting search criteria outlined below.

Toxicology studies identified at licensing were reviewed for ovarian histology and reproductive histories of vaccinated female rats.

Clinical safety trials presented at licensing were reviewed for documentation of age at menarche, menstrual patterns pre-vaccine and ongoing patterns post-vaccine. Menarche age is required because cycles are commonly irregular for 12 to 18 months following menarche [13]. Cycle recording and reporting is required since irregularity is the first symptom in $92 \%$ of ovarian failure. Case series of adolescent idiopathic POI after QHPV vaccination and the general population's interval years to diagnosis suggest follow-up of menstrual patterns for more than 5 years in non-hormone-using women to evaluate ovarian health. Documentation for 5 years might only detect 75\% of POI [9]. Young women using hormonal contraception, including long-acting reversible contraceptives (LARCS), cannot contribute to ovarian safety research due to hormone masking. Etonorgestrel implants and progestin-containing intra uterine devices (IUDs) disable ovarian observation for 3 to 5 years. Since the publication of case series of POI/POF, national health departments $[14,15]$ and regulatory bodies $[14,16]$ reference pre-licensing studies as evidencing ovarian safety after QHPV vaccine. To answer the question 'has the ongoing ovarian safety of this vaccine in adolescents been assessed?', clinical licensing studies were analysed regarding enrolments, design, controls, process, appropriate participant selection, follow-up intervals, reporting decisions, and concomitant hormonal usage assessing internal validity and consistency.

Post-licensing safety studies were reviewed in a literature search of QHPV post-marketing safety studies from 2006 to September 15th 2015. Medline, Embase, and Cochrane databases were searched for reports using words 'Gardasil', ' 'quadrivalent human papillomavirus vaccine' and 'safety', 'adverse events', 'adverse drug reactions', 'toxicity', 'vaccine safety' and 'side-effects'. Results were then hand-searched for randomized controlled studies and observational studies with adolescent enrolments using the World Health Organization definition of adolescents as young people between the ages of 10 and 19 years. Safety reviews and metaanalyses of safety data were hand searched for additional references. All identified QHPV vaccine safety studies with adolescent enrolments between 10 and 19 years of age were analysed for recorded menstrual histories or menstruation reports pre- and postvaccine, assessment of menstrual function and capacity to evaluate menstrual function. Studies of women older than 19 years, studies of women with chronic disease and those comparing multiple vaccines were not included.

Vaccine Adverse Event Reporting Systems in USA and Australia were observed from licensing to February 2016.

Safety statements and product information were observed.

\section{Results}

\section{Pre-licensing}

Toxicology Studies: The internal validity [17] of pre-clinical ovary and fertility testing is limited by absent assessment of ovarian structure, absent assessment of ongoing function, and failure to complete vaccination prior to fertility testing.

Rodent toxicology and fertility studies identified at licensing were reviewed for evidence of ovarian structural and cellular integrity and ongoing function post QHPV vaccine. 
Pre-clinical safety studies of QHPV vaccine administered two of the three doses which have comprised the Gardasil ${ }^{\oplus}$ vaccination course to five-week-old virgin rats before testing their ability to conceive $[18,19]$. One of these doses was administered 5 weeks before mating and the second dose was administered 2 weeks before mating. Fertility after the 3-dose vaccination was not tested, since no female rats completed vaccination before mating. Two further vaccine doses were administered: one on gestation day 6 and one on lactation day 7.

No histology report of tested rat ovaries is available. Histology reporting of the tested male gonad was normal [20,21]. The only recorded observation of the tested rodent ovary is a numbering of corpora lutea [22]. There were 366 corpora lutea in 22 unvaccinated rat controls, and 326 corpora present in the 22 rats given two vaccine doses before mating. No further histological information was available under a Freedom of Information Request to the Therapeutic Goods Administration (TGA) of Australia [23].

Young tested rats were euthanized after their first litter. The duration of their reproductive life span, frequency of subsequent litters, litter spacing and subsequent numbers of pups per litter were not assessed.

U.S. Department of Health and Human Services 'Guidance for Industry' research protocols state "Where possible we recommend that you administer the maximum human dose (e.g. 1 human dose equals 1 rabbit dose) regardless of body weight" [24]. Completion of the vaccination course prior to mating is not a requirement. However, incomplete dosing before testing rats' ability to conceive reduces the reliability and generalizability of this vaccine's fertility safety margin for 3-dose vaccinated teenage girls. The pre-licensing conclusion that QHPV vaccine fertility testing gave 'a safety margin of 200-fold by body weight' [25], calculated by dividing human weight by rat weight $(0.25 \mathrm{~kg})$, assumes 3 -dose course fertility testing and assumes the vaccine recipient's weight to be $50 \mathrm{~kg}$. (which is above 50th centile weights [26] in the vaccine target age group of $37 \mathrm{~kg}$ for 11 year-olds and $42 \mathrm{~kg}$ for $12 \mathrm{year}$ olds). The fertility safety margin from pre-licensing toxicology is more accurately represented as 148 to 168 -fold for two vaccine doses when three doses constituted a vaccine course.

A '10 week old repeat dose toxicity study' in BALB/C mice [27] conducted by Merck Sharp and Dohme prior to 2006 is referred to by the European Medicines Agency but not cited. No reproductive outcomes from this study are displayed at licensing by the Food and Drug Administration or referenced by the European Medicines Agency or Therapeutic Goods Administration. This study is not referenced in Product Information discussion of fertility. The TGA were unable to supply this study upon formal request (TGA reply 30/3/2017). This study can no longer be accessed.

Clinical studies: Phase II and Phase III adolescent studies identified as safety studies at licensing [28] by the VRBPAC to the FDA are study protocols V501 007, [29] 016, [30] 018, [31] and 013, [32] 015 [1] respectively. Clinical studies lacked the benefit of generalizable pre-clinical fertility studies to inform study design. Short follow-up intervals, unrecorded menstrual patterns of adolescents, large and unaccounted attrition rates, and improper control principles and representation reduce the internal validity and generalizability of licensing safety trials, particularly in relation to ovarian function.

Protocols 016 and 018: Safety studies of young adolescent girls at licensing were protocols 016 and 018.

Protocol 016 [30] was the first young teen safety study. It was not a randomized controlled trial. Protocol 016 does not meet usual measures for assessing study quality $[17,33,34]$. With its small numbers, safety goal unmet, inadequately explained attrition, short follow-up, subjective investigator decisions and no control, the design, performance, detection and attrition weaknesses [17] of this early Phase II study reduced its capacity to attest to ovarian function.

Participants were aged 10 to 15 years, mean age 12.6 years. The proportion post-menarche is not known. Authors stressed the importance of monitoring safety for 12 months from first vaccination, but of the 494 fully vaccinated young adolescent's girls in this trial 52\% were lost to follow-up and only 240 fully-vaccinated girls remained at 12 months for safety assessment. Loss is mostly attributed to a protocol amendment, 'withdrawn consent' (no reason given) or 'lost to follow-up'. An older cohort of 467 females aged 16 to 23 years were observed for immunogenicity and none were followed up at 12 months for safety assessment. A Vaccine report card (VRC) recorded adverse events (AEs) for 2 weeks after each vaccination and interviews collected adverse events at months 2, 3 and 7. Naturally irregular menses in immediate post menarche months [35] may not be distinguished from ovarian dysfunction by the girl or at interview. Furthermore, primary and secondary amenorrhoea is poorly identifiable in this aged population. Investigators 'were instructed to assign causality on the basis of exposure, time course, likely cause and consistency with the test vaccine's known profile. When this study was conducted between 2002 and 2004, however, the safety profile of the test vaccine was not established. One 13-year-old experienced vaginal haemorrhaging 26 days post dose 2 , and 42 days post dose 3. This met the study's pre-defined serious adverse event (SAE) criteria (life-threatening or resulting in persistent or significant disability or incapacity or death) and responded to hormone treatment. The reporting physician judged it 'probably vaccine related' but subsequent gynaecological opinion attributed it to an unspecified pre-existing condition missed at enrolment. While this trial's safety objective of 12 month follow-up was not met, longer follow-up would have been needed to attest to ovarian safety. The median interval from menstrual disorder to diagnosis of POF is two years [9]. Five years follow-up would be needed to detect 75\% of cases of ovarian failure in non-hormone-using women [9]. 
Protocol 018 [31] was the second young adolescent safety study. Its small number of fully vaccinated participant girls reduced its ability to measure less common events. Its 11.9 year mean participant age, unrecorded menarche status, unreported menstrual patterns and VRC design had minimal ability to detect or attest to ovarian function. Misrepresentation of the placebo as 'saline' in the study abstract compromises the integrity of the study report and is continued in safety reviews [36,37] of the vaccine and in licensing approval [38].

Protocol 018 was the final study assessing QHPV vaccine safety in young adolescents. It was a randomized, double blind, placebocontrolled study. Protocol 018 was relied upon at QHPV vaccine licensing as a safety study, and cited in Gardasil ${ }^{\circledR}$ Product Information as a safety study. Its primary safety hypothesis is that 'a 3-dose regimen of quadrivalent HPV vaccine is generally well-tolerated in adolescents and pre-adolescents'. The published study abstract wrongly states a 'saline placebo' was used. This trial's control is later described as containing 'identical components to those in the vaccine with the exception of HPV L1 VLPs and aluminium adjuvant'. The placebo contained $50 \mu \mathrm{g}$ polysorbate 80 (polyoxyethylene sorbitan mono-oleate, 'TWEEN 80 '), 35 $\mu \mathrm{g}$ borax, $9.56 \mathrm{mg}$ sodium chloride and $0.78 \mathrm{mg}$ L-histidine. This trial fully vaccinated 587 girls, with $52.3 \%$ aged 9 to 12 and mean age 11.9 years. An additional 301 girls received 3 doses of polysorbate 80 etc as the control. An unknown proportion of girls were post-menarche and capable of reporting menstrual cycle patterns or abnormalities. The study prompted for 'pre-specified adverse experiences' which did not include menstrual dysfunction. Serious adverse events (associated with hospitalization, disability, death, life-threatening, or requiring surgical/medical correction to avoid such an outcome) were also recorded. VRCs recorded adverse experiences within 14 days of each vaccination. Health interviews at 12 months from third vaccination might not have determined menstrual abnormalities from developing ovulatory patterns. One girl in 018 also developed dysfunctional uterine bleeding severe enough to constitute a SAE. This was judged not vaccine-related by the investigator. No basis for this decision is given and no cause was cited [31]. The subjective assignation of vaginal haemorrhage as a vaccine related event by the 016 investigator and not by the 018 investigator indicates the inconsistency of investigator decisions. Although 563 vaccinated girls completed follow-up in this trial, the majority aged less than 12 years could not contribute to ovarian safety observation. As the European Medicines Agency observed 'for some separate age cohorts [the database] might not sufficiently detect possibly precluded unexpected adverse reactions'[27]. While 500 participant enrolment design is in the mid-range of acceptable numbers of enrolments for phase II studies, the complete absence of the vaccine target age group in phase III studies places added safety reliance on the smaller number of phase II participants who were post menarche.

018 study design breached the control principles that a placebo should lack the substance being tested and have no known pharmacologic properties [39]. The placebo's vaccine constituent polyoxyethylene sorbitan mono-oleate is not inert. It had prior established rat ovary toxicity at all injected doses over a tenfold range [40]. There is no dose-response curve suggesting safe injected dosages of polysorbate 80 ('Tween 80') for the developing ovary. In prior research, untreated control rats' mean oestrus cycle length was 4.3 days from 10 to 18 weeks of age. For rats injected as newborns with $1 \%$ polysorbate 80 , oestrus cycle length increased to 13.2 days at 10 weeks of age, 14.0 days at 14 weeks and 11.6 days at 18 weeks of age. This prolongation was statistically significant. Polysorbate 80 treated rats also had statistically significant decreased ovarian weights compared with untreated controls. Injected rats also showed uterine vascular abnormalities. This study concludes '4-day administration of Tween 80 to female rats during the period crucial for the development and function of reproductive organs accelerates the maturation of these organs' This QHPV vaccine component was a poor choice for inclusion in its safety trial placebo, and a confounding control to assess the vaccine's ovarian safety. The Center for Biologics Evaluation and Research (CBER) inaccurately states "protocol 018 provides saline placebocontrolled safety data for subjects 9 to 15 years. This is of particular interest because the other studies used alum placebo as a safety comparison" [41].

References to a 'saline' control persist throughout subsequent FDA licensing [42] from page 12 to page 426 and in the subsequent extension study of this trial, [43] and continue in Product Information [44] (PI).

Protocols 007, 013, and 015: Protocols 007, 013 and 015 safety trials did not enroll participants from the current preferred vaccine target age group but did enroll 16 year-olds, mean age was 20 years. These trials' internal validity, capacity and generalizability to infer ovarian safety are reduced by absent menstrual histories pre- and post- vaccine, fortnight reporting limitations, all placebos incorrectly identified as per corrected Australian PI at this author's request, [45] hormonal masking in the majority's use of hormonal contraception, restricted definitions of 'Serious Adverse Events', short follow-up and subjective reporting of adverse events by investigators.

Phase II Protocol 007 [29] fully vaccinated 256 women aged 16 to 23 years with the current Gardasil ${ }^{\circledR}$ product. It was a randomized, double blind, placebo-controlled study. As in all older women studies 'participants were required to use effective contraception throughout the trial' and $75 \%$ to $82 \%$ of women used hormonal contraception within 15 days of any vaccination [46]. Hormonal masking significantly prevented ovarian health observation post vaccination. The study states aluminium hydroxyphosphate sulphate formed the control, but does not identify the $50 \mu \mathrm{cg}$ polysorbate 80 also present in each of three placebo doses. Follow-up for 36 months does not include menstrual records. The validity, generalizability and capacity of this trial to evidence ovarian safety are very limited.

Phase III Protocol 013 [32] 'Future 1' study enrolled 16 to 24 year-olds, mean age 20.3 years. 2543 women completed vaccination. 
Journal of Immunology and Infectious Diseases

The safety objective was 'to demonstrate that a three dose regimen of the quadrivalent HPV is generally well tolerated' [47]. This was a randomized, double blind, placebo-controlled efficacy study. It identifies its placebo as the same 'aluminum-containing' placebo as protocol 007, the vaccines 'amorphous aluminium hydroxyphosphate sulphate adjuvant'. It fails to identify the $50 \mu c g$ polysorbate 80 in its control[45]. A VRC recorded adverse events within 2 weeks of each vaccination, with gynaecology health concerns and SAEs solicited at 2, 3, 6 and 7 months, and further SAEs collected for the follow-up period. 55\% completed 24 month follow-up from 3rd vaccination and 3\% completed follow-up 30 months from 3rd vaccination. The 58\% of participants using hormonal contraception at the start of the study, rose to $68 \%$ to $83 \%$ of participants in the two sub-studies of protocol 013 [48], affecting observation of ovarian function. Participants were required to use effective contraception to month 7.

Unreported menstrual cycles, high participant hormonal usage, VRC fortnight reporting limitations, reliance on restricted classifications of 'SAEs' (excluding altered menses) to assess ongoing safety and long term follow-up less than that usually needed for diagnosis of premature ovarian insufficiency [9] render this study unable to attest to ovarian safety. A review of QHPV vaccine pre-licensing safety studies reports: 'new medical conditions were not considered adverse events if they occurred post month 7 or were not considered by the investigator to be vaccine-related'[36]. This further reduced capacity to detect ovarian dysfunction and progressive insufficiency which could only surface after contraception was permitted to cease at 7 months. When the CBER requested analysis of autoimmune conditions across the entire safety database the sponsor replied 'there were additional subjects with new medical conditions that were not reported in the Clinical Study Reports for 011 and 012 (within protocol 103). These included two subjects with amenorrhea' [49]. This evidences a flaw in the reporting process. New conditions recorded infrequently in a trial of a new vaccine with 'causality assigned by the investigator' may not reach an investigator's threshold of plausibility [14]. Non-reporting of amenorrhoea suggests a detection weakness and bias in assessing the safety of the QHPV vaccine regimen regarding ongoing ovarian function and raises a concern in the overall reporting process of phase III safety trials.

Protocol 015 [1] 'Future II' was a randomized, double blind, placebo-controlled Phase III study. Its primary hypothesis concerned efficacy in reducing high grade intraepithelial neoplasia, but safety was a stated 'primary objective' [50] at licensing. This largest safety study misidentifies an 'aluminum-containing placebo' and fails to identify the vaccine's $50 \mu \mathrm{g}$ polysorbate 80 in each placebo dose [45]. The fully-vaccinated 5916 [51] participants were aged 16 to 24 years (one was 15) with mean age 20 years and more than two thirds using hormonal contraception. Menarche is not recorded. Participants were followed for 30 months average from first vaccination. A small cohort of 448 vaccines and 447 controls formed the 'Detailed Safety Cohort' recording non-serious adverse events (NSAEs) for two weeks post injection and longer term SAEs. Menstrual cycles, were not recorded through the trial. Other cohorts recorded SAEs only in this interval. Cycle disturbances, oligomenorrhea and amenorrhea do not signal as SAEs. Small numbers in the 'Detailed Safety Cohort', a misrepresented placebo with potential for ovary toxicity, exclusion of the peri-pubertal vaccine-targeted group, failure to record menstrual cycles and hormonal usage in over two thirds of participants disabled this study from establishing ovarian safety. There were no cases of POI/POF reported in vaccinated or control subjects during clinical trials.

SAE definitional limitations in Phase III studies introduced a detection weakness for detecting ovarian dysfunction. This bias was increased by the Phase III design decision that 'new medical conditions were not considered adverse events if they occurred post month 7' [36] when hormone usage could cease. Follow-up times were too brief for most population POF/POI diagnoses. Aluminium has been connected to possible associations with autoimmune and other ovarian damage, $[3,52,53]$ is not inert, and may also be a confounding placebo choice The 'Autoimmune Inflammatory Syndrome Induced by Adjuvants' [3] has been presented as a cause of auto-immune ovarian damage leading to premature menopause. The first case series had symptoms consistent with this presentation [54] and anti-ovarian antibodies were detected in one 15-year-old. It has been considered that a genetic predisposition may favor induction of autoimmune pathology initiated by aluminium. The presentation of siblings with premature menopause and no prior family history would be consistent with this aetiology. Rat ovary structure has also been 'disrupted' after aluminium chloride ingestion and reduced expression of follicle stimulating hormone and luteinizing hormone receptors has been observed [52]. The decision to include the vaccine's aluminium adjuvant and unidentified polysorbate 80 surfactant in placebos of all phase III safety studies reduced their integrity, validity [52] and generalizability as controlled studies. This error also continues throughout FDA licensing references.

Following notification to the TGA of placebo misrepresentation in PI (20/6/2015), TGA Deputy Secretary [55] replied (31/8/2015) Merck Sharp and Dohme Australia Pty Ltd had been subsequently requested to amend Gardasil ${ }^{\oplus}$ PI. Commonwealth Serum Laboratories adverse reaction review in PI 4/2/2016 [56] now states 'both aluminium and non-aluminium containing placebos contained the same minor ingredients $(0.78 \mathrm{mg}$ histidine and $50 \mu \mathrm{g}$ polysorbate 80$)$ as Gardasil'. This conflicts with the placebo description in Future I, Future II and protocol 007 studies and with PI [57] in other countries. Since Merck Sharp and Dohme clearly value the use of a saline placebo and wished to impute one had been used, it is unclear why a saline placebo was not used in any safety trials of this wholly new virus like particle vaccine.

\section{Post-licensing}

Controlled Studies: There were no controlled safety studies of QHPV vaccine which enrolled adolescent women and reported on menstrual function. 
Observational studies: One observational study reported menstrual function. A retrospective analysis [58] of 75 patients consecutively presenting to a syncope unit for investigation of autonomic dysfunction after QHPV vaccine over 3 years 6 months between May 2011 and December 2014 recorded menstrual irregularities post vaccine. Those not reporting symptoms within 2 months of vaccination or with chronic diseases were excluded, leaving 53 young women in this study. Adolescents from age 12 were included and the mean age was 23 years. The frequencies of the most common symptoms in this group were recorded. Of those not using oral contraception $48 \%$ recorded irregular periods following vaccination. This small study focusing on autonomic dysfunction is the only QHPV vaccine post-licensing study which reported on menstrual function following vaccination.

\section{Safety reviews}

Safety reviews and meta-analyses added no new safety study references. No reviews considered ongoing menstrual function, or acknowledged missing research in reported menarche and menstrual function. No safety reviews analysed studies' internal validity with respect to selected placebos.

Five post-marketing studies were most commonly cited as establishing QHPV vaccine safety due to their impressive number of participants $[59,60]$ length of follow-up $[43,61]$ or scope [62] but did not meet search criteria for this safety review. These controlled studies did not have the capacity to detect menstrual evidence of ovarian dysfunction.

1. A 2012 post-licensure commitment[59] sought to evaluate safety 'during the course of routine clinical care'. This retrospective, observational, risk interval cohort study reviewed presentations to Emergency Departments and hospitalizations in 44,000 fullyvaccinated women aged 9 to 26 years. Altered menstrual cycles, oligomenorrhoea and amenorrhoea do not require emergency departments or hospitalization. POI if present would still be present at interval comparison. Its analysis[63] for 16 specified autoimmune conditions did not include POF/POI.

2. A register-based cohort study of vaccine-associated SAEs in 997,585 10-17 year-old girls[60] in Sweden and Denmark 2006 to 2010 measured incident hospital diagnosed autoimmune, neurological and thromboembolic events. Menstrual cycle abnormalities were not included in the pre-specified conditions sought and do not require emergency medicine and hospital care.

3. The 14 year follow-up extension study of V501-015 [61] 4800 participants included only SAEs [64] and vaccinated its placebo cohort. Ovarian dysfunction is not included in SAE definitions.

4. The 10 year follow-up of V501-018 [43] included only SAEs and pregnancies. Early vaccination of the placebo cohort removed its control.

5. The Vaccine Safety Datalink (VSD) is a collaboration of managed care organizations [65]. It can provide information on 9 million people annually, collecting weekly information from hospitals, emergency departments and outpatient clinics. It has reported on pre-specified adverse events [62] after QHPV vaccine, but not menstrual dysfunction, POI or POF. The VSD utilizes 'rapid cycle analysis' [65] of pre-selected vaccine adverse events compared with the expected occurrence in the background population. This requires knowledge of background rates or incidence rates from an unvaccinated cohort. A VSD study is planned to investigate POI and QHPV vaccine. Since age-specific POI/POF rates are unknown in young adolescents and vaccination is widespread this method might utilize the higher background rates of POI/POF in older and dissimilar populations. Since the target age of 12 to 13 years [66] is also the common menarche age group, the pre-vaccine age group cannot form a control. Hormone contraceptive usage will disqualify controls. The only valid control group would be an un-vaccinated, non-hormone using cohort of age-matched adolescents. The planned VSD POF study will need to identify a post-vaccine observation window or 'risk period' of well over 5 years, given the $25 \%$ of POF who take more than 5 years to diagnosis [9] and the variable duration of pre-menopausal symptoms. There is no reason to suppose a shorter menopausal prodrome in adolescents than in mature women. Concurrence of the vaccine target age group with peri-menarche makes observation difficult. Since less than $40 \%$ of women with secondary amenorrhoea contact a doctor, [10] and long-acting reversible contraception is increasing [67] VSD analysis of this diagnosis may not be generalizable. Long term effects of vaccination are more difficult to assess by this method [68] due to biases in the unvaccinated comparator group.

\section{Adverse Event Reporting Systems}

The USA Centers for Disease Control and Prevention (CDC) and the FDA maintain the Vaccine Adverse Event Reporting System (VAERS). This database was observed in August 2011, August 2013 and on February $24^{\text {th }} 2016$.

'Amenorrhoea' notifications post Gardasil ${ }^{\circledR}$ listed in VAERS were reviewed, excluding those with recent OCP usage or breastfeeding. 123 amenorrhoea notifications are recorded (24/2/2016). Only one (VAERS 486279-1) had a Follicle Stimulating Hormone (FSH) result ('elevated at 72' consistent with POI/POF, units not given). Only one reports an Anti Mullerian Hormone (AMH) level (VAERS 526361-1). Her notifying endocrinologist reports AMH as 'low' and diagnosed 'sudden ovarian insufficiency' in this 16-year-old girl. A third simply records 'irregular hormones'. This highlights three problems in VAERS. First, the database has not adopted POI terminology, so POI may list as 'amenorrhoea', or under several other headings. Second, the diagnosis of the 120 amenorrhoeic girls without lab work is unknown. Third, amenorrhoea is under-investigated in primary care and in VAERS 
notifications. Clinical data is brief: 'Cessation of menstruation followed vaccination with Gardasil ${ }^{\circledR}$ after history of regular periods' (VAERS 562101-1). Under 'Ovarian Disorder' VAERS lists 'low ovarian reserve' (Case ID 528889) without follow-up or lab results. 'Ovarian Failure' lists four cases, aged 14 and 15 years at onset, other ages not given.

'POF' cases are poorly recorded, but suggest 13 cases with ages supplied for six at 15, 16, 18 and two 'in her twenties' one with comment 'did not realize until off of birth control and trying to conceive'.

A further VAERS database problem may be deficient record-keeping. VAERS 342035 age 13 was recorded as diagnosed premature menopause August 2011 after regular periods since menarche, age 11. VAERS 342035 however, appears only as 'amenorrhoea' with an 'unknown outcome' in August 2013. Altered notifications compromise the ability of an adverse event reporting system to detect early warning signals and generate hypotheses for future research.

Adverse event notifications are mostly of events occurring soon after vaccination. Outcomes with delayed onset or not recognized as associated with vaccination can have significantly lower reporting sensitivities [69] and are affected by 'health care workers awareness of particular adverse events'. Passive reporting relies on voluntary effort. The under-reporting of serious and severe adverse drug reactions is significant, with the median under-reporting rate assessed at $94 \%$ [70].

\section{Safety statements}

The CDC and FDA reviewed notifications to VAERS of POF and premature menopause as POI following QHPV4 vaccine after distribution of 80,000,000 doses. Patterns of development of ovarian failure were analysed [71] from June 2006 to September 2015. VAERS diagnostic categories do not include POI but use the Medical Dictionary for Regulatory Activities (medDRA) category 'POF' [72]. Since no consistent patterns were discerned and the number of these cases notified after QHPV vaccine fell within the expected range of cases, it was concluded that no association between QHPV vaccine and POI was in evidence. Unfortunately, however, the background incidence and pattern variation of rare idiopathic premature ovarian insufficiency development in 13 to 16 year-olds is un-researched and unknown. The 123 amenorrhoea notifications discussed above should bear equal analysis. Gonadotropin measurement has been recommended by some in all young women with amenorrhoea [73]. Three months or more amenorrhoea has been identified as the presenting symptom in 58\% of young women with POF [9]. Given the identification of only one FSH level and one AMH level among these amenorrhoea notifications, and the evidenced delay to both presentation and diagnosis of POF in primary care, the evaluation of POF in 80,000,000 vaccines should have included consideration of reported adverse events symptoms consistent with POF.

Notifications to VAERS, furthermore, rely on voluntary reporting and are incomplete. Reporting occurs for only a proportion of suspected adverse events [69] and voluntary reports in passive systems come from populations of unknown size. It is not possible therefore to determine the incidence of these adverse events from VAERS reports or to assess causality [74]. Patterns, too, will be significantly obscured by adolescent hormonal therapy prescribed for acne, contraception and especially for cycle management, delaying symptom development and diagnosis as in the second case series [4]. Adverse event reporting efficiency [74] may be reduced by the inconsistent nature of POI symptoms [9], lack of diagnostic event proximity to vaccination [70] and decreased notifications as distance from vaccination increases, under-investigation and 5 year delay to POF diagnosis, [9] failure of 60\% of women with amenorrhoea to consult a medical practitioner [10], ovarian function masking by subsequent hormonal contraception with OCP or long acting reversible contraceptives, the mingling of POI presentations with common adolescent menstrual cycle changes, discouragement of this notification by health authorities' condemnation as a 'myth' and 'not biologically plausible' [14], and statements from licensing authorities that QHPV vaccine has already been researched in relation to the ovary and found safe [71]. Any hindrance of adverse event reporting efficiency renders QHPV vaccine vial distribution a poor contributor to establishing this vaccine's safety for the developing ovary.

Due to accepted VAERS analysis limitations, the Vaccine Safety Datalink (VSD) is currently conducting a study of any potential link to POI cases. A VSD will face similar limitations, however, to VAERS analysis with no known background age-specific incidence rate of young teenage idiopathic POI or unvaccinated cohort comparator. Meanwhile, the CDC subsequent safety statement informs providers and consumers: 'before Gardasil was licensed, its safety was extensively studied in clinical trials. These studies found no difference in amenorrhea (when a woman of reproductive age doesn't have a period) between women who got Gardasil compared to women who received a placebo (a shot with no medicine in it)' [71]. While this statement accords with USA PI [57] and FDA licensing [42] it does not accord with safety research trials. The Immunize Australia Program incorrectly states 'studies of high doses of HPV vaccine in female and male rats showed no evidence of infertility' [14]. High dose HPV vaccines were not used to test female rat fertility. Formal instruction to Australian immunization providers that any link between POI and QHPV vaccine is a 'myth' and not 'biologically plausible' [14] may negatively impact vaccine event 'reporting efficiency' [74] and pattern detection.

\section{Discussion}

Clinician case reports cannot establish causality. They form the lowest evidentiary level but may be the first indication of a causal connection to an adverse outcome. Notifications and case series of rare unexpected but significant, adverse outcomes [75] of new vaccines stimulated structured review of existing research. 
Pre-licensing safety studies of QHPV vaccination cannot attest to ovarian safety in adolescents due to issues of design, process, detection and internal validity. No controlled post-licensing safety studies reported on menstrual function in the vaccinated adolescent. In consideration of possible autoimmune sequelae [76] or cumulative toxicology, an appropriate length of risk interval considering induction time and lead time together with known delays in presentation and in correct diagnosis would need to have been observed. The only study reporting on menstrual function post QHPV vaccine was an observational study in which $48 \%$ of girls and young women investigated for syncopal events reported irregular cycles following vaccination. No conclusions can be drawn from this study which was conducted on women presenting to a syncope unit and involved relatively small numbers of only 55 women. The safety of the adolescent ovary after QHPV vaccination has not been assessed.

Case series can alert us to rare, unexpected or delayed adverse events. They provide valid input to pharmacovigilance by presenting first warnings of possible safety signals and stimulate further research. Although it is not known whether QHPV vaccine is linked with POI in young teens, structured review of safety research demonstrates a connection cannot yet be ruled out. It also suggests that pharmaceutical disregard for accurate placebo description may indicate flaws in peer-review, in the process of pharmaceutical company evaluation of its own products [77] and in licensing requirements.

Ovarian safety assessment of QHPV vaccine is currently wholly reliant on case notifications. These may not always be well received [78] but must be met with a scientific response and well-designed research. The Medical Director of the National HPV Vaccination program in Australia references past safety statements and the 200,000,000 vaccine doses produced as evidencing vaccine ovarian safety [79]. Providers and consumers are recommended the 'Skeptical Raptor' blogsite 'providing evidence about vaccine safety', indicating an ideological response in preference to a scientific one. The 'excellent' blogsite insists saline-controlled safety research has been done and suggests the large post-marketing QHPV vaccine studies evidence ovarian safety while denigrating reporting/ reporters of possible vaccine adverse events. Meanwhile, a 'serious decline in general medical practitioners reporting adverse drug reactions' has been observed in the TGA's latest performance report [80]. A low level of consumer awareness of available reporting systems had previously been demonstrated [81].

Reliance on the number of vaccine vials manufactured may not adequately establish vaccine ovarian safety.

In the context of multiple published cases of established idiopathic adolescent premature ovarian insufficiency and notifications of this adverse event specifically related to QHPV vaccination, the dearth of research into ovarian safety post QHPV vaccine from initial female rat studies through to the present day is of some concern.

\section{Conclusion}

Vaccines are the most life-saving intervention of all time. It has been rightly said that we cannot live without vaccines, so public confidence in vaccines and in regulatory bodies must be maintained by rigorous and responsive research. While the QHPV vaccine is an excellent development which can reduce the incidence of infection with oncogenic HPV strains by $70 \%$, the possible link to idiopathic adolescent POI is not a trivial matter. Research into this phenomenon urgently needs to be undertaken and requires appropriate menstrual cycle recording and analysis for a minimum of 5 years, with investigation as indicated in vaccinated and unvaccinated cohorts. It is not known whether this vaccine may be contraindicated in some girls. Hormonal contraception may render these girls invisible until choosing to start a family.

\section{Acknowledgments}

Sally Toms, Kathleen O’Meley, Dr. Harvey RG Ward, Dr. Don Radford, Helen Wyborn, Bernadette Morrison, Harriet Radford. There are no competing interests and this work has no funding source.

\section{References}

1. Future II Study Group (2007) Quadrivalent vaccine against human papillomavirus to prevent high-grade cervical lesions. N Engl J Med 356: $1915-27$.

2. Australian Government of Health (2016) Immunize Australia Program. Retrived on 31st May \& 19th November 2016.

3. Colafrancesco S, Perricone C, Tomljenovic L, Shoenfeld Y (2013) Human Papilloma Virus Vaccine and Primary Ovarian Failure: Another Facet of the Autoimmune/Inflammatory Syndrome Induced by Adjuvants. Am J Reprod Immunol 70: 309-16.

4. Little DT, Ward HRG (2014) Adolescent Premature Ovarian Insufficiency Following Human Papillomavirus Vaccination: A Case Series Seen in General Practice. J Inve Med High Impact Case Rep 1-12.

5. Little DT, Ward HR (2012) Premature ovarian failure 3 years after menarche in a 16-year-old girl following human papillomavirus vaccination. BMJ Case Reports. doi:10.1136/bcr-2012-006879.

6. Little DT (2013) Human Papillomavirus Vaccine and the Ovary: the Need for Research. Proceedings of the 18th World Congress on Controversies in Obstetrics, Gynecology and Infertility. 2013. Vienna: Monduzzi Editoriale.

7. Little DT (2013) Human papillomavirus vaccine and possible effect on ovarian function, in 23rd World Congress on Controversies in Obstetrics, Gynecology and Infertility 2016: Melbourne Australia.

8. Baker VL (2013) Primary ovarian insufficiency in the adolescent. Curr Opin Obstet Gynecol 25: 375-81.

9. Alzubaidi NH, Chapin HL, Vanderhoof VH, Calis KA, Nelson LM (2002) Meeting the needs of young women with secondary amenorrhoea and spontaneous premature ovarian failure. Obstet Gynecol 99: 720-5. 
10. Münster K, Helm P, Schmidt L (1992) Secondary amenorrhoea: Prevalence and medical contact - a cross-sectional study from a Danish county. Br J Obstet Gynecol 99: 430-3.

11. Coulam CB, Adamson SC, Annegers JF (1986) Incidence of Premature ovarian failure. Obstet Gynecol 67: 604-6.

12. Ellenberg SS (2001) Safety considerations for new vaccine development. Pharmacoepidemiol Drug Saf 10: 411-5.

13. DeCherney A, Nathan L, Goodwin TM, Laufer N (2007) Current Diagnosis and Treatment Obstetrics and Gynecology (10 ${ }^{\text {th }}$ Edn) Lange McGraw-Hill, USA. 14. Myths and Realities (2013) A Guide for Providers. Aust Gov Dept of Health and Ageing.

15. Quadrivalent HPV vaccine - Fact Sheet. Accessed on March 26 ${ }^{\text {th }}, 2017$.

16. Wathion N (2016) EMA response to Nordic Cochrane letter on HPV vaccines - maladministration, Denmark.

17. Juni P, Altman D, Egger M (2001) Assessing the Quality of Controlled Clinical Trials. BMJ 323: 42-6.

18. Wise, L.D., et al., (2008) Lack of effects on fertility and developmental toxicity of a quadrivalent HPV vaccine in Sprague-Dawley rats. Birth Defects Rese B Dev Reprod Toxicol 83: 561-72.

19. Intramuscular developmental toxicity and immunogenicity study in rats with postweaning evaluation. Extract - Study no. TT\#03-703-0 (CTD Module 4, volumes 1-3). Table A-6.

20. Wise LD, Pauley CJ, Michael B, Wolf JJ (2010) Lack of effects on male fertility from a quadrivalent HPV vaccine in Sprague-Dawley rats. Birth Defects Res B Dev Reprod Toxicol 89: 376-81.

21. TGA (2011) Australian Public Assessment Report for Human Papillomavirus Quadrivalent Vaccine February. 2011.

22. Extract study no. TT\#03-703-0 (CTD Module 4, volumes 1-3) Study summary for non-clinical study report 'Intramuscular developmental toxicity and immunogenicity study in rats with post-weaning evaluation'. 2005, Merck \& Co.

23. FOI Request 001-1112 in relation to GARDASIL testing and complied with in the public interest Sept 2011. Application not displayed on TGA FOI website listing due to 'commercially sensitive nature'. 2011.

24. Guidance for Industry: Considerations for Developmental Toxicity Studies for Prevention and Therapeutic Vaccines for Infectious Disease Indications. Center for Biologics Evaluation and Research February 2006.

25. Wise LD, Wolf JJ, Kaplanski CV, Pauley CJ, Ledwith BJ (2008) Lack of effects on fertility and developmental toxicity of a quadrivalent HPV vaccine in SpragueDawley rats. Birth Defects Res B Dev Reprod Toxicol 83: 561-72.

26. Oates KK, Currow, Hu W (2001) Child Health A practice manual for general practice. Maclennan \& Petty.

27. EMEA (2006) Scientific Discussion Gardasil, INN-Human Papillomavirus Vaccine [Types 6,11,16,18].

28. FDA Government (2006) Background Document GARDASIL (Tm) Human Papillomavirus Quadrivalent Vaccine VRBPAC Meeting May 2006, USA.

29. Villa LL, Costa RL, Petta CA, Andrade RP, Ault KA, et al. (2005) Prophyllactic quadrivalent human papillomavirus (types 6, 11, 16 and 18) L1 virus-like particle vaccine in young women: a randomized double-blind placebo-controlled multicentre phase II efficacy trial. Lancet Oncol 6: 271-8.

30. Block SL, Nolan T, Sattler C, Barr E, Giacoletti KE, et al. (2006) Comparison of the immunogenicity and reactogenicity of a quadrivalent human papillomavirus (types 6,11,16 and 18) virus-like particle vaccine in male and female adolescents and young adult women. Pediatrics 118: 2135-45.

31. Reisinger KS, Block SL, Lazcano-Ponce E, Samakoses R, Esser MT, et al. (2007) Safety and persistent immunogenicity of quadrivalent human papillomavirus Types 6, 11, 16 and $18 \mathrm{~L} 1$ virus-like particle vaccine in preadolescents and adolescents. Pediatr Infect Dis J 26: 201-9.

32. Garland SM, Hernandez-Avila M, Wheeler CM, Perez G, Harper DM, et al. (2007) Quadrivalent vaccine against human papillomavirus to prevent anogenital diseases. N Engl J Med 356: 1928-43.

33. Cook DJ, Sackett DL, Spitzer WO (1995) Methodologic Guidelines for Systematic Reviews of Randomized Control Trials in Health Care from the Potsdam Consultation on Meta-Analysis. J Clin Epidemiol 48: 167-71.

34. Jadad AR, Moore RA, Carroll D, Jenkinson C, Reynolds DJ, et al. (1996) Assessing the Quality of Reports of Randomized Clinical Trials: Is Blinding Necessary? Controlled Clinical Trials 17: 1-12.

35. Menstruation in girls and adolescents:using the menstrual cycle as a vital sign. ACOG, 2015.

36. Block SL, Brown DR, Chatterjee A, Gold MA, Sings HL, et al. (2010) Clinical trial and post licensure safety profile of a prophylactic human papillomavirus (types 6, 11, 16 and 18) L1 virus-like particle vaccine. Pediatr Infect Dis J 29: 95-101.

37. Bonanni P, Cohet C, Kjaer SK, Latham NB, Lambert PH, et al. (2010) A summary of post-licensure surveillance initiatives for GARDASIL/SILGARD(R) Vaccine 28: 4719-30.

38. Miller NB (2006) Clinical Review of Biologics License Application for Human Papillomavirus 6, 11, 16, 18 L1 VirusLike Particle Vaccine. (S. cerevisiae) (STN 125126 Gardasil) Center for Biologics Evaluation and Research Food and Drug Administration, USA.

39. Emanuel EJ, Grady CC, Crouch RA, Lie RK, Miller FG, et al. (2011) The Oxford Textbook of Clinical Research Ethics. The Ethics of Placebo-controlled Trials 2011 In: Medical Law Review, Oxford University Press Inc: New York, USA.

40. Gajdová M, Jakubovsky J, Války J (1993) Delayed effects of neonatal exposure to Tween 80 on female reproductive organs in rats. Fd Chem Toxic 31: 183-90.

41. Miller NB (2006) Clinical Review of Biologics License Application for Human Papillomavirus 6, 11, 16, 18 L1 VirusLike Particle Vaccine. (S. cerevisiae) (STN 125126 Gardasil) Center for Biologics Evaluation and Research Food and Drug Administration P: 330.

42. Miller NB (2006) Clinical Review of Biologics License Application for Human Papillomavirus 6, 16, 18 L1 VirusLike Particle Vaccine. (S. cerevisiae) (STN 125126 Gardasil) Center for Biologics Evaluation and Research Food and Drug Administration, USA.

43. Iverson O-E (2016) Long-term extension study of GARDASIL (R) in adolescents; results through month 96 in On behalf of the Protocol 018 investigators., University of Bergen: Department of Clinical Science.

44. Medsafe Government NZ (2010) Product Information GARDASIL ${ }^{\oplus}$, Quadrivalent Human Papillomavirus (Types 6, 11, 16, 18) Recombinant vaccine, WPC-V501-I-022011.

45. Product Information Gardasil ${ }^{\oplus}$ WPC-V501-1-022011 [Quadrivalent Human Papillomavirus (Types 6, 11, 16, 18) Recombinant vaccine], Australia.

46. Miller NB (2006) Clinical Review of Biologics License Application for Human Papillomavirus 6, 11, 16, 18 L1 VirusLike Particle Vaccine. (S. cerevisiae) (STN 125126 Gardasil) Center for Biologics Evaluation and Research Food and Drug Administration P: 216. 
47. Miller NB (2006) Clinical Review of Biologics License Application for Human Papillomavirus 6, 11, 16, 18 L1 VirusLike Particle Vaccine. (S. cerevisiae) (STN 125126 Gardasil) Center for Biologics Evaluation and Research Food and Drug Administration P: 125.

48. Miller NB (2006) Clinical Review of Biologics License Application for Human Papillomavirus 6, 11, 16, 18 L1 VirusLike Particle Vaccine. (S. cerevisiae) (STN 125126 Gardasil) Center for Biologics Evaluation and Research Food and Drug Administration P: 143.

49. Miller NB (2006) Clinical Review of Biologics License Application for Human Papillomavirus 6, 16, 18 L1 VirusLike Particle Vaccine. (S. cerevisiae) (STN 125126 Gardasil) Center for Biologics Evaluation and Research Food and Drug Administration P: 198.

50. Miller NB (2006) Clinical Review of Biologics License Application for Human Papillomavirus 6, 16, 18 L1 VirusLike Particle Vaccine. (S. cerevisiae) (STN 125126 Gardasil) Center for Biologics Evaluation and Research Food and Drug Administration P: 47.

51. Miller NB (2006) Clinical Review of Biologics License Application for Human Papillomavirus 6, 16, 18 L1 VirusLike Particle Vaccine. (S. cerevisiae) (STN 125126 Gardasil) Center for Biologics Evaluation and Research Food and Drug Administration P: 58.

52. Fua Y, Jiab FB, Wanga Y, Songa M, Liuc SM, et al. (2014) Effects of sub-chronic aluminium exposure on rat ovaries. Life Sciences 100: 61-6.

53. Triff A, V Igna, E Dumitrescu (2008) The consequences of chronic exposure to aluminium sulphate on fundamental markers of the reproductive function in female rats. Vetrerinary Medicine 65: 457-61.

54. Perriconea C, Colafrancescoa S, Mazora RD, Sorianoa A, Agmon-Levina N, et al. (2013) Autoimmune/inflammatory syndrome induced by adjuvants (ASIA) 2013: Unveiling the pathogenic, clinical and diagnostic aspects. J Autoimmun 47: 1-16.

55. Skerritt J (2015) TGA Formal Communication re Gardasil Product Information Safety Trial Data. Accessed on August $31^{\text {st }} 2015$.

56. Merck, Sharpe, and Dohme, Product Information Gardasil (R). 201617.

57. Full Prescribing Information for GARDASIL (R). Accessed on September $09^{\text {th }}, 2016$.

58. Brinth L, Theibel AC, Pors K, Mehlsen J (2015) Suspected side effects to the quadrivalent human papilloma vaccine. Dan Med J 62: 1-5.

59. Klein NP, Hansen J, Chao C, Velicer C, Emery M, et al. (2012) Safety of human papillomavirus vaccine administered routinely to females. Arch Pediatr Adolesc Med 166: 1140-8.

60. Arnheim-Dahlstrom L, Pasternak, Svanströmet H, Sparén P, Anders Hviid (2013) Autoimmune, neurological and venous thromboembolic events after immunization of adolescent girls with quadrivalent human papillomavirus vaccine in Denmark and Sweden: cohort study. BMJ 347: 15906.

61. Nygard M (2013) Long-term effectiveness and immunogenicity of Gardasil ${ }^{\circ}$ in the Nordic countries, Eurogin Florence Italy.

62. Gee J, Naleway A, Shui I, Baggs J, Yin R, et al. (2011) Monitoring the safety of quadrivalent human papillomavirus vaccine: Findings from the Vaccine Safety Datalink. Vaccine 29: 8279-84.

63. Chao C, Klein NP, Velicer CM, Sy LS, Slezak JM, et al. (2011) Surveillance of autoimmune conditions following routine use of quadrivalent human papillomavirus vaccine. J Int Med 271: 183-203.

64. US Food and Drug Administration. Regulatory definition 21 CFR \#314.80 Postmarketing reporting of adverse drug experiences. Retrived on August $6^{\text {th }}$, 2015.

65. Baggs J, Gee J, Lewis E, Fowler G, Benson P, et al. (2011) The vaccine safety datalink: a model for monitoring vaccine immunization safety. Pediatrics 127: 54553.

66. Australian Government Department of Health (2016) Immunize Australia Program, Australia.

67. Guttamacher Institute (2015) Contraceptive Use in the United States, USA.

68. Andrews NJ (2002) Staatistical assessment of the association between vaccination and rare adverse events post-licensure. Vaccine 20: 549-53.

69. Rosenthal S, Chen R (1995) The reporting sensitivities of two passive surveillance systems for vaccine adverse events. Am J Public Health 85: 1706-9.

70. Hazell L, Shakir SA (2006) Under reporting of adverse drug reactions: a systematic review. Drug Safety 29: 385-96.

71. CDC (2016) Centers for Disease Control Frequently asked questions about HPV vaccine safety. Accessed on Sept $12^{\text {th }}, 2016$.

72. Medical Dictionaries for Regulatory Activities (2017) Premature ovarian failure, USA.

73. Rebar RW, Connolly HV (1990) Clinical Features of young women with hypergonadotropic amenorrhoea. Fertil Steril 53: 804-10.

74. Varricchio F, Iskander J, Destefano F, Ball R, Pless R, et al. (2004) Understanding vaccine safety information from the vaccine adverse event reporting system. Pediatr Infect Dis J 23: 287-94.

75. Agha R, Rosin RD (2010) Time for a new approach to case reports. It J Surg 1: 1-3.

76. Chao C, Jacobsen S (2012) Evaluation of autoimmune safety signal in observational vaccine safety studies. Hum Vaccin Immunother 8: 1302-4.

77. Agnell M (2008) Industry-sponsored clinical research: a broken system. JAMA 300: 1069-71.

78. Hawkes D, Buttery J (2016) Human Papillomavirus Vaccination and Primary Ovarian Insufficiency: An Association Based on Ideology Rather Than Evidence. Curr Opin Obstet Gynecol 28: 70-2.

79. Brotherton J, Hawkes D, Saville M (2016) Vaccination questions need patient response. MJA Insight 2016. Accessed on $26^{\text {th }}$ March 2017.

80. Woodhead M (2016) Concern over fall in GP adverse drug effect reports, in Australian Doctor, Australia.

81. Robertson J, Newby D (2013) Low awareness of adverse drug reaction reporting systems: a consumer survey. MJA 199: 684-6. 


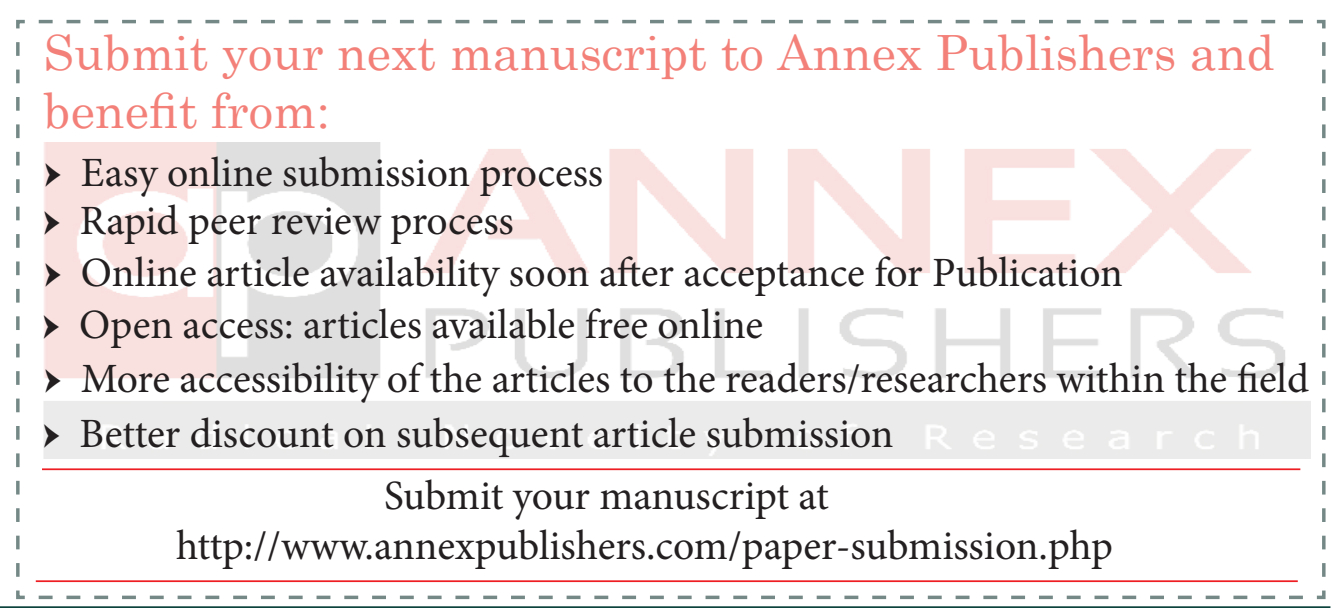

